\title{
Impact of stepwise introduction of esophagojejunostomy during laparoscopic total gastrectomy: a single-center experience in Japan
}

\author{
Daiki Yasukawa, Tomohide Hori, Yoshio Kadokawa, Shigeru Kato, Takafumi Machimoto, Toshiyuki Hata, \\ Yuki Aisu, Maho Sasaki, Yusuke Kimura, Yuichi Takamatsu, Tatsuo Ito, Tsunehiro Yoshimura
}

Tenriyorodusoudanjyo Hospital, Tenri, Japan

Abstract

Background The number of laparoscopic gastrectomies performed in Japan is increasing with the development of laparoscopic and surgical instruments. However, laparoscopic total gastrectomy is developing relatively slowly because of technical difficulties, particularly in esophagojejunostomy.

Methods We retrospectively reviewed 83 patients with early gastric cancer in the upper portion of the stomach who underwent laparoscopic total gastrectomy between April 2007 and March 2016. We classified the patients into three periods, mainly on the basis of the esophagojejunostomy procedures performed: first period, various conventional procedures based on the physicians' choice $(n=14)$; second period, transoral method $(n=51)$; and third period, fully intracorporeal technique $(n=18)$. We evaluated the clinical impact of a stepwise introduction of unfamiliar new methods during laparoscopic total gastrectomy.

Results Between the first and second periods, there were significant differences in the blood loss volume, number of harvested lymph nodes, frequency of conversion to open surgery, and postoperative hospital stay. The number of harvested lymph nodes was significantly higher in the third than in the second period, with no detriment to other intraoperative or postoperative factors.

Conclusion The use of a unified surgical method for esophagojejunostomy seems to be the key to a successful and advantageous laparoscopic total gastrectomy. Stepwise introduction of a wellestablished technique of esophagojejunostomy during laparoscopic total gastrectomy will benefit patients, as shown, for example, by the higher number of dissected lymph nodes in the present study. However, a protracted learning curve is required.

Keywords Esophagojejunostomy, gastric cancer, laparoscopic total gastrectomy

Ann Gastroenterol 2017; 30 (4): 1-7

\section{Introduction}

Gastric cancer is one of the most common causes of cancer-related death worldwide. Particularly in Eastern Asian countries, such as Japan, the mortality rate associated with gastric cancer remains very high [1]. The detection rate of early

Department of Gastrointestinal and General Surgery,

Tenriyorodusoudanjyo Hospital, Tenri, Japan

Conflict of Interest: None

Correspondence to: Tomohide Hori, $\mathrm{PhD}, \mathrm{MD}$, Department of Gastrointestinal and General Surgery, Tenriyorodusoudanjyo Hospital, 200 Mishima-cho, Tenri 632-8552, Japan, Tel.: +81 74363 5611, Fax: +81 74363 1530, e-mail: horitomo@tenriyorozu.jp

Received 4 January 2017; accepted 12 April 2017; published online 11 May 2017

DOI: https://doi.org/10.20524/aog.2017.0157 gastric cancer (EGC) has recently been increasing [2]. Thus, it is reasonable that early detection and screening programs for gastric cancer and subsequent surgical treatment, including appropriate lymph node (LN) dissection, have been successfully developed in high-risk areas such as Eastern Asia [2,3].

Each country has its own health insurance system; the Japanese government employs a universal health insurance system. Therefore, novel surgical procedures in Japan are not authorized until they receive a listing in the health insurance system by the governmental council. The first report of laparoscopy-assisted distal gastrectomy (LADG) was documented in 1994 [4], and laparoscopic gastrectomy (LG) was approved by the Japan governmental council in 2002. LADG has since developed markedly [5,6] and has been widely performed for treatment of EGC in recent years. LG in the treatment of EGC has many advantages, including better cosmesis, less postoperative pain, less operative stress, earlier postoperative recovery, earlier meal ingestion, a shorter hospital stay, and a higher quality of life [5-8]. The safety and 
efficacy of LG for EGC have been demonstrated in several clinical studies $[5,9,10]$. Moreover, the current study clearly demonstrates that LADG for advanced gastric cancer is also feasible, safe, and effective in selected patients [11].

However, laparoscopic total gastrectomy (LTG) has developed relatively slowly because of technical difficulties, particularly in esophagojejunostomy (i.e. esophagojejunal reconstruction [EJR]). Extracorporeal EJR through a minilaparotomy has been performed during LTG. However, it is often difficult to complete the anastomosis though this minilaparotomy because of the narrow window for insertion of the anvil head and instruments [12]. Side-to-side anastomosis or the overlap method for intracorporeal EJR during LTG has refined complicated reconstructive procedures and provided impressively satisfactory outcomes $[13,14]$, although LTG with these novel techniques may require an advanced skill set.

We have been performing LTG for patients with EGC in the upper portion of the stomach since April 2007 and have thus developed an impression regarding the safety and feasibility of LTG for EGC [12]. Our institution appears to have gone through three eras of anastomotic techniques. We retrospectively evaluated 83 patients who underwent LTG, according to the anastomotic patterns of EJR. We investigated intraoperative factors, oncological findings, the postoperative course, morbidity, and mortality in each of the periods and herein we discuss the effects of a stepwise introduction of unfamiliar new methods during LTG.

\section{Patients and methods}

\section{Patients}

We retrospectively reviewed 83 consecutive patients who underwent LTG with Roux-en-Y (RY) reconstruction at our institution between April 2007 and March 2016. Preoperative staging was based on the findings of upper gastrointestinal endoscopy, upper gastrointestinal series, and enhanced computed tomography. Based on the findings of the preoperative examinations, gastric cancers exhibiting either deep invasion into the proper muscle layer or LN metastasis were excluded from LG. All patients were preoperatively diagnosed with EGC located in the upper portion of the stomach, and the depth of tumor invasion was limited to the submucosa without LN metastasis. This study design was approved by the Institutional Review Board of Tenriyorodusoudanjyo Hospital and performed in accordance with the Declaration of Helsinki.

\section{Surgical procedures of LTG}

The patient was placed in an open-leg position. The operator stood on the right side of the patient, the first assistant stood on the left side, and the laparoscopist stood between the patient's legs. After carbon dioxide pneumoperitoneum of $8-10 \mathrm{mmHg}$ had been established though the umbilical port, four operating ports were placed in the upper abdomen.
A flexible laparoscope was then introduced via the umbilical port; a three-dimensional laparoscopic system was employed (Endoeye Flex 3D, EVIS EXERA III; Olympus, Tokyo, Japan). During LTG, dissection of the LNs was D1 plus dissection, as defined by the Japan Gastric Cancer Treatment Guideline, $4^{\text {th }}$ ed., in 2014 [15]. In our institution, a total of twelve surgeons performed this surgery, under two supervisors. Hence, our surgical procedures were performed in a unified way.

\section{Transoral method}

We performed EJR by the transoral method, which involves intracorporeal circular stapling with a transorally inserted anvil (OrVilTM; Covidien, Mansfield, MA, USA). According to the manufacturer's instructions, this anvil tube was introduced transorally into the esophagus and a smaller hole was created on the esophageal stump. A 4-cm longitudinal mini-laparotomy incision was made between the epigastrium and umbilicus. The mini-laparotomy wound was retracted and protected using a wound retraction device (Alexis Wound Retractor; Applied Medical, Rancho Santa Margarita, CA, USA). The stomach was then extracted through the minilaparotomy. A side-to-side jejunojejunostomy was made in the fashion of RY reconstruction, and a $45-\mathrm{cm}$-long section of jejunum accompanied by the mesenterium (so-called RY limb) was prepared for subsequent EJR. The actual surgical procedures are shown schematically in Fig. 1.

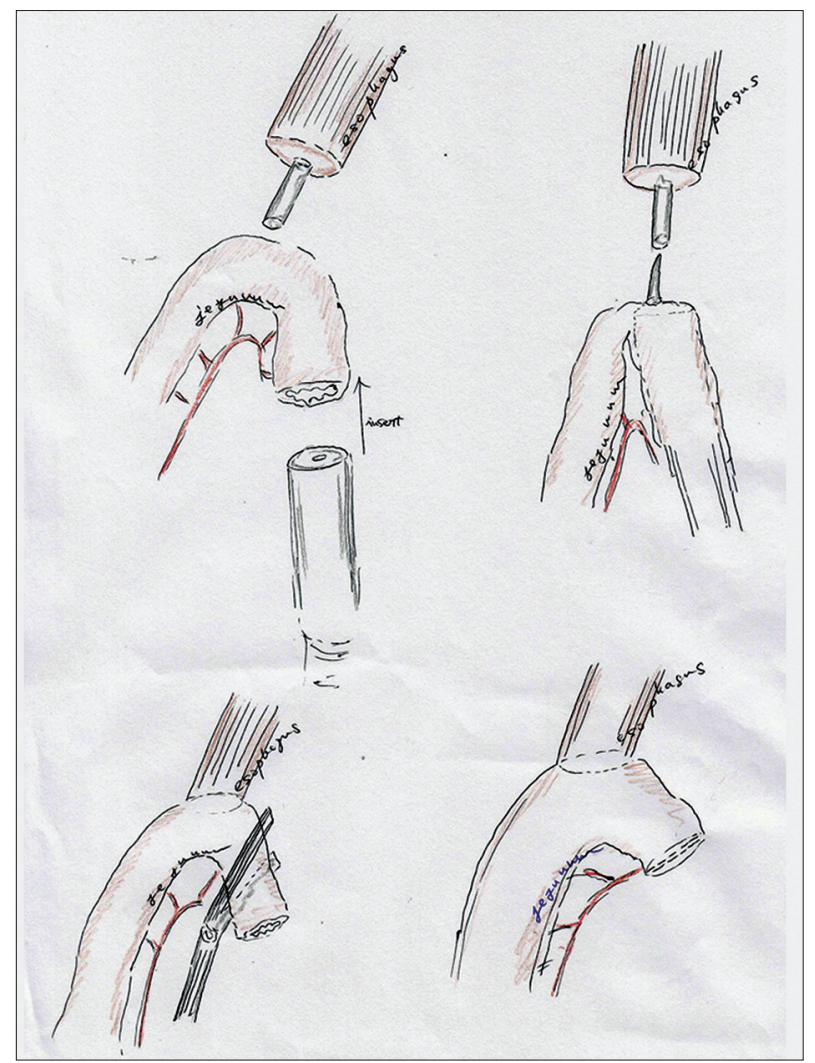

Figure 1 Actual procedures of transoral method 


\section{Fully intracorporeal technique}

To complete the functional end-to-end anastomosis for fully intracorporeal EJR, we employed a side-to-side anastomosis. A minimal incision (only $4.5 \mathrm{~cm}$ long) was made in the umbilical portion; this was used for extraction of the stomach and for an extracorporeal formation of a side-to-side jejunojejunostomy prior to EJR. The same above-described RY limb was made. The entry hole on the jejunum was placed on the opposite side of the mesenterium and was made as small as possible. Pneumoperitoneum was then resumed after the wound retraction device had been sealed using a surgical glove. All further procedures for EJR were completed laparoscopically. The entry hole on the esophageal stump was also made as small as possible, and the tip of the nasogastric tube appeared through this entry hole. A laparoscopic stapler (TristapleTM [purple cartridge, $45 \mathrm{~mm}$ ], EndoGIATM; Covidien) was inserted via a suitable working port. The stapler jaw was first applied to the jejunum, and the other jaw was then inserted into the esophageal stump with adequate guidance from the nasogastric tube. The stapler was then used to grasp the esophagus and jejunum without any involvement of the surrounding tissues and fired. The whole layer of the combined entry hole was lifted by fixation sutures and closed by a two-step stapling technique. A couple of sutures remained on the mesenterium to close the abnormal hiatus and prevent twisting of the EJR. The actual surgical procedures are shown schematically in Fig. 2.

\section{Classification}

We classified a total of 83 patients into 3 periods, according to the main procedures performed for EJR: first period, various conventional procedures based on the physicians' choice ( $\mathrm{n}=14)$ (April 2007 to March 2009); second period, transoral method ( $\mathrm{n}=51$ ) (April 2009 to July 2014); and third period, fully intracorporeal technique $(n=18)$ (August 2014 to March 2016).

\section{Statistical analysis}

Data are presented as mean \pm standard deviation. The Mann-Whitney $U$ test and $\chi^{2}$ test were used for comparison of unpaired continuous or discontinuous variables between two groups. Statistical calculations were performed using StatView software, Version 5.0 (SAS Institute Inc., Cary, NC, USA). Values of $\mathrm{P}<0.05$ were considered statistically significant.

\section{Results}

\section{Patients' profiles}

We checked whether the patients underwent preoperative endoscopic submucosal dissection because of concern that

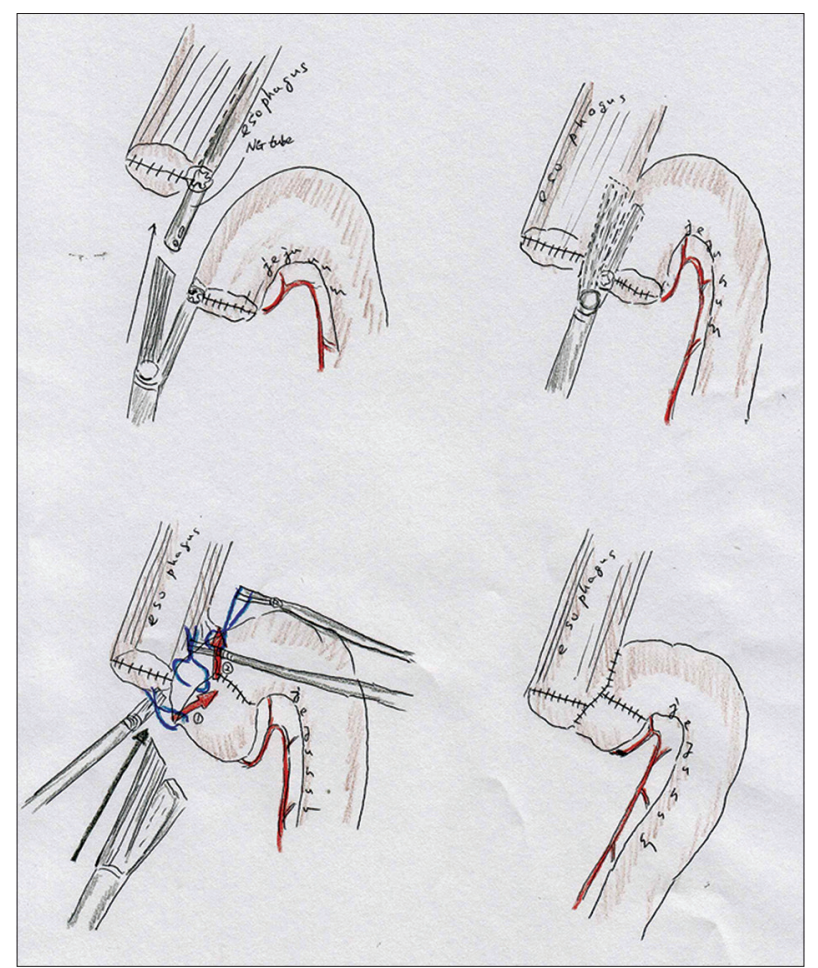

Figure 2 Actual procedures of fully intracorporeal technique

reactive/inflammatory LN swelling may have been detected in the imaging studies and/or intraoperative findings. The patients' profiles are summarized in Table 1. There were no significant differences in age, sex, body mass index, or performance of preoperative endoscopic submucosal dissection, between the first and second periods or between the second and third periods.

\section{Procedures of EJR in each period}

The actual procedures of the esophagojejunal anastomoses performed in each period are summarized in Table 2. In the first period, various EJR procedures were performed, based on the physicians' choice. In the second period, the transoral method was mainly employed in a total of 48 patients (94.1\%). In the third period, the fully intracorporeal technique was employed in 17 of 18 patients (94.4\%).

\section{Intraoperative factors}

Intraoperative factors, such as the operation time, blood loss, number of harvested LNs, and conversion to open surgery are shown in Table 3. In the comparison between the first and second periods, we observed no significant difference in the operation time; however, there were significant differences in blood loss, number of harvested LNs, and frequency of conversion to open surgery. In the comparison between the second and third periods, there were no significant differences 
Table 1 Patients' profiles in each period

\begin{tabular}{lccccc}
\hline Parameter & $1^{\text {st }}$ period $(\mathrm{n}=14)$ & $2^{\text {nd }}$ period $(\mathrm{n}=51)$ & $3^{\text {rd }}$ period $(\mathrm{n}=18)$ & $1^{\text {st }}$ vs. $2^{\text {nd }}$ period & $2^{\text {nd }}$ vs. $3^{\text {rd }}$ period \\
\hline Age $(\mathrm{y})$ & $66.4 \pm 12.1$ & $67.8 \pm 9.4$ & $65.3 \pm 12.6$ & $\mathrm{NS}$ & $\mathrm{NS}$ \\
Male $(\%)$ & $12(85.7)$ & $34(66.7)$ & $15(83.3)$ & $\mathrm{NS}$ & NS \\
BMI & $23.4 \pm 2.5$ & $22.7 \pm 2.5$ & $23.1 \pm 3.0$ & $\mathrm{NS}$ & NS \\
History of ESD (\%) & $4(28.6)$ & $14(27.5)$ & $3(16.7)$ & NS & NS \\
\hline
\end{tabular}

BMI, body mass index; ESD, endoscopic submucosal dissection; NS, not significant

$\underline{\text { Table } 2 \text { Surgical procedures of esophagojejunal anastomoses, in each period }}$

\begin{tabular}{lccc}
\hline Procedure & $1^{\text {st }}$ period $(\mathrm{n}=14)$ & $2^{\text {nd }}$ period $(\mathrm{n}=51)$ & $3^{\text {rd }}$ period $(\mathrm{n}=18)$ \\
\hline Transoral method, $\mathrm{n}(\%)$ & $1(7.1)$ & $48(94.1)$ & $0(0)$ \\
Fully intracorporeal technique, $\mathrm{n}(\%)$ & $3(21.4)$ & $0(0)$ & $17(94.4)$ \\
Conventional anastomosis after conversion to open surgery, $\mathrm{n}(\%)$ & $5(35.7)$ & $2(3.9)$ & $1(5.6)$ \\
Others, $\mathrm{n}(\%)$ & $5(35.7)$ & $1(2.0)$ & $0(0)$ \\
\hline
\end{tabular}

Table 3 Intraoperative factors in each period

\begin{tabular}{|c|c|c|c|c|c|}
\hline Factor & $1^{\text {st }}$ period $(n=14)$ & $2^{\text {nd }}$ period $(n=51)$ & $3^{\text {rd }}$ period $(n=18)$ & $1^{\text {st }}$ vs. $2^{\text {nd }}$ period & $2^{\text {nd }}$ vs. $3^{\text {rd }}$ period \\
\hline Operation time (min) & $363 \pm 94.3$ & $346.1 \pm 52.7$ & $348.4 \pm 53.5$ & NS & NS \\
\hline Blood loss* $(\mathrm{mL})$ & $85(26-380)$ & $34(10-556)$ & $35(10-750)$ & 0.0001 & NS \\
\hline Number of harvested LNs & $29.4 \pm 9.5$ & $40.8 \pm 16.7$ & $51.1 \pm 19.0$ & 0.0216 & 0.0499 \\
\hline Conversion to open surgery (\%) & $5(35.7)$ & $2(3.9)$ & $1(5.5)$ & 0.0007 & NS \\
\hline $\begin{array}{l}\text { Conversion to open surgery due } \\
\text { to difficulty in EJR (\%) }\end{array}$ & $5(35.7)$ & $1(2.0)$ & $0(0.0)$ & 0.0001 & NS \\
\hline
\end{tabular}

${ }^{*}$ Data were shown as median and range

LN, lymph nodes; NS, not significant; EJR, esophagojejunal reconstruction

in the operation time, blood loss, or frequency of conversion to open surgery, whereas a significant difference was identified in the number of harvested LNs. Surprisingly, the number of harvested LNs was clearly improved (increased). As regards the difficulty of EJR causing conversion to open surgery, there was a significant difference between the first and second periods, but not between the second and third periods. Technical difficulty in EJR was the major reason for conversion to open surgery in the first period. Unexpected intraoperative findings (one case in the second period) and severe obesity (one case in the third period) were other reasons. Although conversion is known to result in increased operative time, a longer operative time was not observed during the first period in our study, despite the significantly higher frequency of conversion due to technical difficulties with EJR and the greater blood loss.

\section{Oncological findings}

The pathological stages according to the seventh edition of the Union for International Cancer Control (UICC) are shown in Table 4. The distribution of tumors was similar in each period.

\section{Anastomotic complications}

All complications associated with EJR are detailed in Table 5. There were no significant differences between the first and second periods or between the second and third periods. Although conversion is known to result in a higher complication rate from the anastomosis, an increased anastomotic complication rate was not observed in our study during the first period, despite the significantly higher frequency of conversion due to technical difficulties with EJR.

\section{Postoperative course}

Postoperative complications greater than grade III according to the Clavien-Dindo classification are shown in detail in Table 6. There were no significant differences in the incidence of severe complications between the first and second periods or between the second and third periods. The postoperative hospital stay in the second period was shorter than that in the first period, but there was no difference between the second and third periods (Table 6). Only one hospital death, due to severe pneumonitis, was observed in the first period (Table 6). 
Table 4 Oncological findings in each period

\begin{tabular}{lccccc}
\hline TNM pathological stage (\%)* & $1^{\text {st }}$ period $(\mathrm{n}=14)$ & $2^{\text {nd }}$ period $(\mathrm{n}=51)$ & $3^{\text {rd }}$ period $(\mathrm{n}=18)$ & $1^{\text {st }}$ vs. $2^{\text {nd }}$ period & $2^{\text {nd }}$ vs. $3^{\text {rd }}$ period \\
\hline I A & $10(71.4)$ & $41(80.4)$ & $14(77.8)$ & NS & NS \\
I B & $2(14.3)$ & $4(7.8)$ & $1(5.6)$ & NS & NS \\
II B & $1(7.1)$ & $5(9.8)$ & $3(16.7)$ & NS & NS \\
II B & $0(0.0)$ & $1(2.0)$ & $0(0.0)$ & NS & NS \\
III A & $1(7.1)$ & $0(0.0)$ & $0(0.0)$ & NS & NS \\
III B/III/IV & $0(0.0)$ & $0(0.0)$ & $0(0.0)$ & NS & NS \\
\hline
\end{tabular}

${ }^{*}$ According to the $7^{\text {th }}$ edition UICC staging

NS, not significant

Table 5 Complications related with esophagojejunal anastomoses, in each period

\begin{tabular}{lccccc}
\hline Complication & $1^{\text {st }}$ period $(\mathrm{n}=14)$ & $2^{\text {nd }}$ period $(\mathrm{n}=51)$ & $3^{\text {rd }}$ period $(\mathrm{n}=18)$ & $1^{\text {st }}$ vs. $2^{\text {nd }}$ period & $2^{\text {nd }}$ vs. $3^{\text {rd }}$ period \\
\hline Stenosis at EJR, Grade II & 0 & 0 & 1 & NS & NS \\
Leakage at EJR, Grade III & 1 & 2 & 0 & NS & NS \\
Total & 1 & 2 & 1 & NS & NS \\
\hline
\end{tabular}

${ }^{*}$ According to Clavien-Dindo classification

EJR, esophagojejunal reconstruction; NS, not significant

Table 6 Postoperative course in each period

\begin{tabular}{|c|c|c|c|c|c|}
\hline Postoperative course & $1^{\text {st }}$ period $(n=14)$ & $2^{\text {nd }}$ period $(n=51)$ & $3^{\text {rd }}$ period $(n=18)$ & $1^{\text {st }}$ vs. $2^{\text {nd }}$ period & $2^{\text {nd }}$ vs. $3^{\text {rd }}$ period \\
\hline \multicolumn{6}{|l|}{ Complications $(\%)^{*}$} \\
\hline Postoperative bleeding & $1(7.1)$ & $2(3.9)$ & $0(0.0)$ & NS & NS \\
\hline Pancreatic fistula & $0(0.0)$ & $1(2.0)$ & $0(0.0)$ & NS & NS \\
\hline Obstructive ileus & $1(7.1)$ & $0(0.0)$ & $1(5.6)$ & NS & NS \\
\hline Leakage of EJR & $1(77.1)$ & $2(3.9)$ & $0(0.0)$ & NS & NS \\
\hline Others & $1(7.1)$ & $0(0.0)$ & $0(0.0)$ & NS & NS \\
\hline Total & $4(28.6)$ & $5(9.8)$ & $1(5.6)$ & NS & NS \\
\hline \multicolumn{6}{|l|}{ Hospital stay } \\
\hline Postoperative day ${ }^{* *}$ (day) & $15.5(12-157)$ & $13.0(9-153)$ & $12.0(9-34)$ & 0.0332 & NS \\
\hline \multicolumn{6}{|l|}{ Mortality } \\
\hline Hospital death (\%) & $1(7.1)$ & $0(0.0)$ & $0(0.0)$ & NS & NS \\
\hline
\end{tabular}

${ }^{*}$ Categorized over grade III according to Clavien-Dindo classification, ${ }^{* *}$ Data were shown as median and range

EJR, esophagojejunal reconstruction; NS, not significant

\section{Factors affected by stepwise introduction of EJR}

The frequency of conversion to open surgery due to the technical difficulty of EJR was clearly greater only in the first period, and the postoperative hospital stay was longer in the first period (Tables 3 and 6). These results seem to reflect a learning curve.

A simple question arises. What factors were affected by the stepwise introduction of EJR during LTG? Overall, the number of harvested LNs clearly increased in a stepwise fashion (Table 3) with no detriment to other intraoperative, oncological, or postoperative factors (Tables 3-6).

\section{Discussion}

With the development of laparoscopic apparatus and surgical instruments, the number of LG procedures is currently increasing in Japan. LG has many advantages in comparison with open gastrectomy, such as improved cosmesis, less postoperative pain, less operative stress, earlier postoperative recovery, earlier meal ingestion, a shorter hospital stay, and a higher quality of life [5-8]. However, LG has a protracted learning curve [16], and the 2014 Japan Gastric Cancer Treatment Guideline, $4^{\text {th }}$ ed., therefore recommends that LG is enrolled in clinical research [15]. We introduced LTG for 
the treatment of EGC in April 2007; to date, 83 patients have undergone LTG at our institution. The anastomotic procedure of EJR is one of the most difficult surgical techniques, and we thus employed various EJR procedures during LTG in the first period of the present study. There were no significant differences in the patients' backgrounds between the first and second periods (Table 1). However, the frequency of conversion to open surgery was clearly greater in the first period, with the main reason for conversion being the technical difficulty of EJR; notably, however, the postoperative complications associated with EJR showed no differences (Tables 3 and 5). These data in the first period support the previous opinion that a protracted learning curve is required for stable anastomoses during LTG $[17,18]$. We have a clear impression that EJR requires advanced skill, although the stepwise introduction of EJR in the second and third periods seemed to be stable (Tables 3, 5, and 6).

Some studies have reported the short-term safety and feasibility of EJR using the transoral method [19-21]. Anastomotic stenosis after LTG occurs more frequently when the transoral method is used [22,23]; nevertheless, our own results showed no significant difference in the complications of EJR by the transoral method with circular stapling compared with the fully intracorporeal technique using linear staplers. The fully intracorporeal technique for EJR during LTG is safe and feasible, and a wide lumen causes less anastomotic stricture [13]. There are controversial opinions as to the best method for EJR during LTG [24-26]. Some surgeons have reported that the fully intracorporeal technique is advantageous for shortening the operative time and hospital stay compared with the transoral method [24], although other surgeons have reported that the transoral method shortens the operative time adequately [25]. Conversely, the safety and feasibility of the overlap method, another technique of fully intracorporeal EJR with linear stapling during LTG, has also been reported [14,27]. However, to determine which EJR procedure is the most feasible technique during LTG, well-designed and carefully considered studies are needed in the near future.

We have unified the procedure of EJR during LTG using the transoral method and fully intracorporeal technique; after the unification of the surgical methodology in the second and third periods some advantages were confirmed (Tables 3 and 6). Unification of the surgical method for EJR seems to be the key for successful and advantageous LTG. Furthermore, in the comparison between the second and third periods, the number of harvested LNs was clearly improved in a stepwise fashion, with no detriment to other intraoperative, oncological, or postoperative factors. Stepwise introduction of EJR techniques during LTG is likely to have oncological benefits for patients with EGC. One possible explanation for this is that the EJR procedures become more simplified with introduction of the fully intracorporeal technique in LTG; this allows LN dissection, one of the most important factors in LTG for gastric cancer, to be performed more aggressively. A laparoscopic approach for the surgical treatment of EGC in the upper portion of stomach is still under consideration, because of its technical demands and the consequent prolonged learning curve. In addition, many studies have demonstrated that LN clearance is adequate in both the laparoscopic and the open approach and that there is no superiority from an oncologic point of view. Although we understand that no relationship has been established between a higher number of harvested LNs and a more secure performance of EJR, we speculate that confident EJR anastomoses following the protracted learning curve allow a greater degree of leeway for performing more aggressive lymphadenectomy during LTG.

We understand that the introduction of novel methods for EJR during LTG is usually done with reservation. In the present study, however, we carefully introduced well-established and more simplified methods during LTG in a stepwise manner. We would suggest that a stepwise introduction of even unfamiliar methods is reasonable, provided that skilled laparoscopic surgeons have validated these new methods. Surgeons should not hesitate to introduce a new but simplified technique of EJR during LTG. New but excellent techniques for EJR during LTG will be developed day by day and will become established in the future.

Of course, this was a retrospective study in a single institution, and this type may be hampered of a number of

\section{Summary Box}

\section{What is already known:}

- GuaThe number of laparoscopic gastrectomies for gastric cancer is currently increasing, and the number of laparoscopic gastrectomies is increasing with the development of laparoscopic and surgical instruments

- Laparoscopic total gastrectomy (LTG) is developing relatively slowly because of technical difficulties, especially in esophagojejunostomy

- The introduction of unfamiliar new methods for esophagojejunostomy during LTG is clinically considered as difficult

\section{What the new findings are:}

- We carefully introduced well-established and more simplified methods during LTG in a stepwise manner

- An institutionally unified surgical method for esophagojejunostomy seems to be a key to successful and advantageous LTG

- Although a protracted learning curve is required, a stepwise introduction of even unfamiliar methods is reasonable if skilled laparoscopic surgeons validate these new methods

- Stepwise introduction of a well-established technique of esophagojejunostomy during LTG will bring benefits, such as a higher number of dissected lymph nodes 
biases and mostly a selection bias. Hence, we understand that the conclusions must be drawn with extreme caution.

In conclusion, stepwise introduction of a well-established technique of EJR during LTG will benefit patients, as shown, for example, by the higher number of dissected LNs in the present study. However, a protracted learning curve is required.

\section{References}

1. Sugano K. Screening of gastric cancer in Asia. Best Pract Res Clin Gastroenterol 2015;29:895-905.

2. Suh YS, Yang HK. Screening and early detection of gastric cancer: east versus west. Surg Clin North Am 2015;95:1053-1066.

3. Katai H. Current status of a randomized controlled trial examining laparoscopic gastrectomy for gastric cancer in Japan. Asian J Endosc Surg 2015;8:125-129.

4. Kitano S, Iso Y, Moriyama M, Sugimachi K. Laparoscopy-assisted Billroth I gastrectomy. Surg Laparosc Endosc 1994;4:146-148.

5. Lee SH, Kim IH, Kim IH, Kwak SG, Chae HD. Comparison of short-term outcomes and acute inflammatory response between laparoscopy-assisted and totally laparoscopic distal gastrectomy for early gastric cancer. Ann Surg Treat Res 2015;89:176-182.

6. Yamashita K, Sakuramoto S, Kikuchi S, et al. Laparoscopic versus open distal gastrectomy for early gastric cancer in Japan: longterm clinical outcomes of a randomized clinical trial. Surg Today 2016;46:741-749.

7. Nunobe S, Kumagai K, Ida S, Ohashi M, Hiki N. Minimally invasive surgery for stomach cancer. Jpn J Clin Oncol 2016;46:395-398.

8. Kim YW, Baik YH, Yun YH, et al. Improved quality of life outcomes after laparoscopy-assisted distal gastrectomy for early gastric cancer: results of a prospective randomized clinical trial. Ann Surg 2008;248:721-727.

9. Shiraishi N, Yasuda K, Kitano S. Laparoscopic gastrectomy with lymph node dissection for gastric cancer. Gastric Cancer 2006;9:167-176.

10. Tanimura S, Higashino M, Fukunaga $Y$, et al. Laparoscopic gastrectomy for gastric cancer: experience with more than 600 cases. Surg Endosc 2008;22:1161-1164.

11. Aurello P, Sagnotta A, Terrenato I, et al. Oncologic value of laparoscopy-assisted distal gastrectomy for advanced gastric cancer: A systematic review and meta-analysis. J Minim Access Surg 2016;12:199-208.

12. Okabe H, Tsunoda S, Tanaka E, Hisamori S, Kawada H, Sakai Y. Is laparoscopic total gastrectomy a safe operation? A review of various anastomotic techniques and their outcomes. Surg Today 2015;45:549-558.

13. Treitl D, Hochwald SN, Bao PQ, Unger JM, Ben-David K. Laparoscopic total gastrectomy with D2 lymphadenectomy and side-to-side stapled esophagojejunostomy. J Gastrointest Surg
2016;20:1523-1529.

14. Kitagami H, Morimoto M, Nakamura K, et al. Technique of Rouxen-Y reconstruction using overlap method after laparoscopic total gastrectomy for gastric cancer: 100 consecutively successful cases. Surg Endosc 2016;30:4086-4091.

15. Japanese Gastric Cancer Association. Treatment. In: Japanese Gastric Cancer Association, Gastric Cancer Treatment Guidelines 2014. $4^{\text {th }}$ ed. Kanehara: Tokyo; 2014, pp. 8.

16. Katai H, Sasako M, Fukuda H, et al; JCOG Gastric Cancer Surgical Study Group. Safety and feasibility of laparoscopy-assisted distal gastrectomy with suprapancreatic nodal dissection for clinical stage I gastric cancer: a multicenter phase II trial (JCOG 0703). Gastric Cancer 2010;13:238-244.

17. Jung H, Son SY, Park YS, et al. The learning curve associated with laparoscopic total gastrectomy. Gastric Cancer 2016;19:264-272.

18. Jeong O, Ryu SY, Choi WY, Piao Z, Park YK. Risk factors and learning curve associated with postoperative morbidity of laparoscopic total gastrectomy for gastric carcinoma. Ann Surg Oncol 2014;21:2994-3001.

19. Lu X, Hu Y, Liu H, et al. Short-term outcomes of intracorporeal esophagojejunostomy using the transorally inserted anvil versus extracorporeal circular anastomosis during laparoscopic total gastrectomy for gastric cancer: a propensity score matching analysis. J Surg Res 2016;200:435-443.

20. LaFemina J, Viñuela EF, Schattner MA, Gerdes H, Strong VE. Esophagojejunal reconstruction after total gastrectomy for gastric cancer using a transorally inserted anvil delivery system. Ann Surg Oncol 2013;20:2975-2983.

21. Liao GQ, Ou XW, Liu SQ, Zhang SR, Huang W. Laparoscopyassisted total gastrectomy with trans-orally inserted anvil $\left(\mathrm{OrVil}{ }^{\mathrm{s}}\right)$ : a single institution experience. World $J$ Gastroenterol 2013; 19:755-760.

22. Inokuchi M, Otsuki S, Fujimori $Y$, Sato $Y$, Nakagawa $M$, Kojima K. Systematic review of anastomotic complications of esophagojejunostomy after laparoscopic total gastrectomy. World J Gastroenterol 2015;21:9656-9665.

23. Zuiki T, Hosoya Y, Kaneda Y, et al. Stenosis after use of the doublestapling technique for reconstruction after laparoscopy-assisted total gastrectomy. Surg Endosc 2013;27:3683-3689.

24. Hiyoshi Y, Oki E, Ando K, et al. Outcome of esophagojejunostomy during totally laparoscopic total gastrectomy: a single-center retrospective study. Anticancer Res 2014;34:7227-7232.

25. Cianchi F, Macrì G, Indennitate G, et al. Laparoscopic total gastrectomy using the transorally inserted anvil $\left(\mathrm{OrVil}^{\mathrm{Tm}}\right)$ : a preliminary, single institution experience. Springerplus 2014;3:434.

26. Shim JH, Yoo HM, Oh SI, et al. Various types of intracorporeal esophagojejunostomy after laparoscopic total gastrectomy for gastric cancer. Gastric Cancer 2013;16:420-427.

27. Yamamoto M, Zaima M, Yamamoto H, Harada H, Kawamura J, Yamaguchi T. A modified overlap method using a linear stapler for intracorporeal esophagojejunostomy after laparoscopic total gastrectomy. Hepatogastroenterology 2014;61:543-548. 